\title{
Rho is a presynaptic activator of neurotransmitter release at pre-existing synapses in C. elegans
}

\author{
Rachel McMullan, Emma Hiley, Paul Morrison, and Stephen J. Nurrish ${ }^{1}$ \\ MRC Cell Biology Unit, MRC Laboratory for Molecular Cell Biology and Department of Pharmacology, University College, \\ London WC1E 6BT, United Kingdom
}

\begin{abstract}
Rho GTPases have important roles in neuronal development, but their function in adult neurons is less well understood. We demonstrate that presynaptic changes in Rho activity at Caenorhabditis elegans neuromuscular junctions can radically change animal behavior via modulation of two separate pathways. In one, presynaptic Rho increases acetylcholine $(\mathrm{ACh})$ release by stimulating the accumulation of diacylglycerol (DAG) and the DAG-binding protein UNC-13 at sites of neurotransmitter release; this pathway requires binding of Rho to the DAG kinase DGK-1. A second DGK-1-independent mechanism is revealed by the ability of a Rho inhibitor (C3 transferase) to decrease levels of release even in the absence of DGK-1; this pathway is independent of UNC-13 accumulation at release sites. We do not detect any Rho-induced changes in neuronal morphology or synapse number; thus, Rho facilitates synaptic transmission by a novel mechanism. Surprisingly, many commonly available human RhoA constructs contain an uncharacterized mutation that severely reduces binding of RhoA to DAG kinase. Thus, a role for RhoA in controlling DAG levels is likely to have been underestimated.
\end{abstract}

[Keywords: RhoA; animal behavior; synaptic vesicle release; DAG kinase; Munc13; C. elegans]

Supplemental material is available at http://www.genesdev.org.

Received July 18, 2005; revised version accepted October 31, 2005.

Extracellular neuromodulators can alter levels of neurotransmitter release at many, if not all, synapses (for reviews, see Miller 1998; Ghijsen and Miriam Leenders 2005). Although many of the molecular components that underlie neurotransmitter release have been characterized (for review, see Sudhof 2004), less is known about how they are regulated by the intracellular signaling pathways that modulate levels of neurotransmitter release.

Rho GTPases are a family of small GTP-binding proteins that regulate a wide variety of signal transduction pathways in all eukaryotic cells (Etienne-Manneville and Hall 2002). The three best studied members of the family-Rho, Rac, and Cdc42-have important roles in the developing nervous system (Govek et al. 2005) and in the regulation of synaptic function, as revealed by behavioral studies. Gene knockouts of several Rho GTPase effectors (PAK, WAVE-1, or LIMK-1) impair learning and memory in mice (Meng et al. 2002, 2004; Soderling et al. 2003), while inhibition of the Rho effector p160ROCK impairs long-term memory in rats (Dash et al. 2004). In humans,

${ }^{1}$ Corresponding author.

E-MAIL s.nurrish@ucl.ac.uk; FAX 44-20-76797805.

Article and publication are at http://www.genesdev.org/cgi/doi/10.1101/ gad.359706. defects in genes for regulators (ARHGEF6, OPHN1) or effectors (LIMK-1, PAK3) of Rho GTPases are associated with mental retardation (Ramakers 2002; Chechlacz and Gleeson 2003).

One possible role for Rho GTPases at synapses is to regulate levels of neurotransmitter release by modulating synaptic vesicle exocytosis. Rac and Cdc42 have been shown to regulate synaptic strength by acting presynaptically in Aplysia (Humeau et al. 2002; Udo et al. 2005), and to induce exocytosis in mammalian secretory cells (Kowluru et al. 1997; Brown et al. 1998). A role for Rho in the regulation of neurotransmitter release is, however, less clear. Potentiation of acetylcholine (ACh) release by lysophosphatidic acid in PC12 cells requires RhoB (Ishida et al. 2004), but, in contrast, adrenalin release from permeabilized PC12 cells has been reported to be independent of RhoA (Wang et al. 2004).

To investigate the role of Rho in regulating neurotransmitter release, we have examined the effect of Rhoinfluencing transgenes on ACh release at the Caenorhabditis elegans neuromuscular junction. The single C. elegans Rho ortholog (RHO-1) is expressed throughout the nervous system (Chen and Lim 1994) and is required for normal cell migration, cell shape regulation, and neurite outgrowth (Zallen et al. 2000; Spencer et al. 
2001; Dalpe et al. 2004). We report here that RHO-1 can enhance ACh release by at least two mechanisms-one dependent on the diacylglycerol (DAG) kinase DGK-1 and one DGK-1-independent. We also show that RHO-1 stimulates synaptic signaling by increasing muscle sensitivity to ACh. Thus, RHO-1 can use three distinct pathways to strengthen synaptic signaling at the C. elegans neuromuscular junction.

\section{Results}

RHO-1 regulates neurotransmitter release at neuromuscular junctions

We used the Clostridium botulinum-derived C3 transferase to inhibit the function of RHO-1 in C. elegans. C3 transferase ADP-ribosylates and inactivates Rho without affecting other Rho family proteins, including Rac and Cdc42 (Aktories and Hall 1989). Expression of N-termi- nally GFP-tagged C3 transferase from an inducible heatshock promoter in adult C. elegans resulted in a major change in behavior, greatly decreasing animal movement as measured by a decrease in body bends per minute and very shallow body bends (Fig. 1A,B). To test whether RHO-1 acts pre- or post-synaptically, we expressed C3 transferase in ACh-releasing neurons, using the promoter for the unc-17 gene, which encodes the ACh transporter in synaptic vesicles (Alfonso et al. 1993). These animals also displayed decreased movement compared with wild-type animals (Fig. 1A,B), confirming that inhibition of endogenous RHO-1 signaling in ACh-releasing cells changes C. elegans locomotion.

To further test the role of RHO-1 in neurotransmitter release, we expressed a constitutively active RHO$1(\mathrm{G} 14 \mathrm{~V})$ mutant from either an inducible heat-shock promoter or the unc-17 promoter. In both cases, expression of RHO-1(G14V) also changed adult behavior, but in this case movement was greatly increased, as measured
Figure 1. RHO-1 signaling alters animal behavior and acetylcholine release. Mutant behavioral assays and transgenic animals were as described in Materials and Methods. (A) Inhibition of endogenous RHO-1 by expression of C3 transferase from heat-shock (hs:) or neuronal (N:) (using the unc-17 promoter) promoters decreased locomotion, whereas expression of a constitutively active RHO$1(\mathrm{G} 14 \mathrm{~V})$ mutant from heat-shock or neuronal promoters increased locomotion. Statistical analysis was performed using a two-tailed $t$-test, and significance is indicated with two asterisks $\left(^{\star \star}, p<0.001\right)$. (B) Locomotion phenotypes of the mutants described in this study. Inhibition of endogenous RHO-1 by heat-shock (hs:C3T) or presynaptic (from the unc-17 promoter, N:C3T) expression of C3 transferase causes flattened sinusoidal tracks compared with wild type. Heat-shock [hs:RHO$1(\mathrm{G} 14 \mathrm{~V})]$ and presynaptic [N:RHO-1(G14V)] expression of RHO-1(G14V) results in exaggerated sinusoidal tracks compared with wild type. $(C-E)$ Synaptic release of endogenous acetylcholine (ACh) was measured by determining the onset of paralysis induced by the acetylcholinesterase inhibitor aldicarb. Expression of C3 transferase from either heatshock (hs:C3 transferase + hs) $(C)$ or the presynaptic unc-17 (N:C3T) (D) promoter delayed the onset of paralysis of animals treated with aldicarb relative to non-heat-shocked animals (hs:C3 transferase hs) or wild-type animals (wt), suggesting a decrease in levels of ACh release. In contrast, expression of the constitutively active RHO-1(G14V) from either heat-shock [RHO-1 $(\mathrm{G} 14 \mathrm{~V})+\mathrm{hs}] \quad(E)$ or unc-17 $(\mathrm{N}: \mathrm{C} 3 \mathrm{~T})(D)$ promoters results in a faster onset of paralysis relative to non-heat-shocked animals [RHO-1(G14V) - hs] or wild-type (wt) animals, suggesting that the normal role of RHO-1 activation is to increase ACh release. Error bars indicate standard error of the mean.

\section{A}

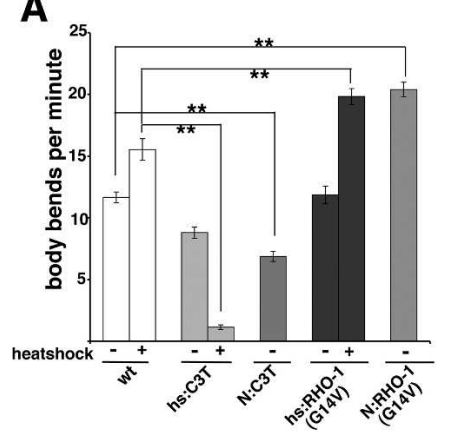

B
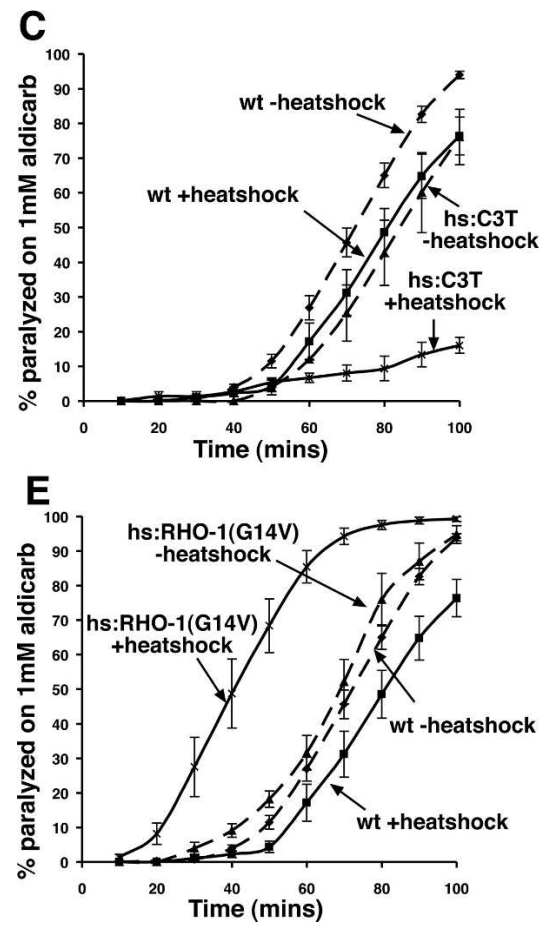

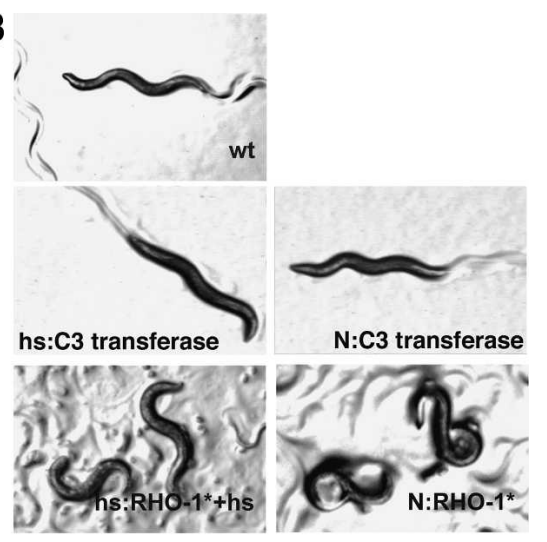

D

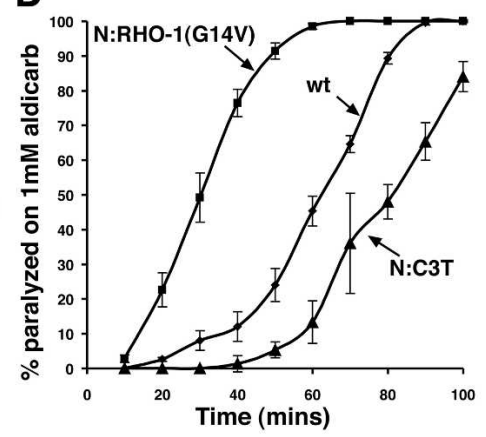


by an increase in body bends per minute, and animals produced exaggerated body bends with increased bend amplitude, producing loopy locomotion (Fig. 1A,B).

To relate the behavioral effects of changing $\mathrm{RHO}-1$ activity to ACh levels, we quantified the responsiveness of animals to the acetylcholinesterase inhibitor aldicarb. Aldicarb prevents the removal of endogenously released ACh, which allows ACh to build up to a level sufficient to hypercontract the body wall muscles and cause paralysis in wild-type animals, but not in mutants that are defective in ACh release (Nonet et al. 1993; Nguyen et al. 1995; Miller et al. 1996). In the presence of aldicarb, heat-shock-induced expression of C3 transferase in adult animals strongly reduced the extent of paralysis compared with both noninduced animals and wild-type animals (Fig. 1C). Presynaptic expression of C3 transferase from the unc-17 promoter also increased resistance to aldicarb treatment compared with wild-type animals (Fig. 1D). In contrast, heat-shock expression of the constitutively active RHO-1(G14V) mutant in adult animals resulted in hypersensitivity to aldicarb (Fig. 1E). RHO$1(\mathrm{G} 14 \mathrm{~V})$ acted presynaptically, as animals expressing activated RHO-1(G14V) in motor neurons from the unc-17 promoter also became paralyzed faster with aldicarb treatment compared with wild-type animals (Fig. 1D). Taken together, these results suggest that activation of RHO-1 enhances ACh release, leading to changes in behavior.

\section{RHO-1 enhances ACh release at pre-existing synapses}

In principle, RHO-1 could increase ACh release either by increasing ACh release at existing synapses, by stimulating the formation of new ACh-releasing synapses, or both. We tested the possibility that RHO-1 increased the formation of new synapses in two ways. First, we looked for changes in neuronal connectivity by assessing GFPlabeled motor neurons and found no obvious morphological differences in wild-type, C3 transferase, and RHO-1(G14V)-expressing motor neurons (Supplementary Fig. 1). Second, we expressed CFP-labeled synaptobrevin (SNB-1::CFP), an integral membrane protein enriched in synaptic vesicles, in the ACh-releasing motor neurons to measure the number of release sites before and after heat-shock-induced expression of the constitutively active RHO-1(G14V). After heat-shock induction of RHO-1(G14V), we saw no change in the number of SNB-1 : CFP puncta compared with noninduced animals (3.2 \pm 0.4 vs. $2.9 \pm 0.2$ puncta per $10 \mu \mathrm{m}$; mean $\pm S E M$; Supplementary Fig. 2). Similarly, expression of RHO$1(\mathrm{G} 14 \mathrm{~V})$ in ACh-releasing neurons (from the unc-17 promoter) did not alter the number of SNB-1::CFP puncta when compared with wild-type controls $(3.2 \pm 0.2$ vs. $3.4 \pm 0.1$ puncta per $10 \mu \mathrm{m}$; mean \pm SEM; Supplementary Fig. 2). We conclude that RHO-1(G14V) exerts its effects by increasing ACh release at pre-existing synapses and not by increasing the number of synapses. C3 transferase was tagged with GFP, and we were unable to visualize SNB-1::CFP puncta; thus, it is possible that inhibition of endogenous RHO-1 may cause a decrease in numbers of release sites.

\section{RHO-1 interacts with DGK-1}

How does RHO-1 enhance neurotransmitter release? Mammalian RhoA has been shown to bind and inhibit the DAG kinase DGK $\theta$ (Houssa et al. 1999; Los et al. 2004). DAG kinases remove the membrane-bound second messenger DAG, which enhances neurotransmitter release in both mice and C. elegans (Lackner et al. 1999; Miller et al. 1999; Brose and Rosenmund 2002; Silinsky and Searl 2003; Brose et al. 2004), and the C. elegans ortholog of DGK $\theta$, DGK-1, acts in motor neurons to inhibit ACh release (Nurrish et al. 1999). Interestingly, animals expressing constitutively active RHO-1(G14V) behave very similarly to both animals exposed to the DAG analog phorbol ester and animals lacking DGK-1 (HajduCronin et al. 1999; Lackner et al. 1999; Miller et al. 1999; Nurrish et al. 1999), whereas animals overexpressing DGK-1 resemble animals expressing C3 transferase. Thus, DGK-1 is a possible target of negative regulation by RHO-1. To test if the interaction between RHO-1 and DGK-1 is conserved in C. elegans, we expressed HAtagged, full-length DGK-1, together with myc-tagged, active RHO-1(G14V), in Cos7 cells. DGK-1 does coimmunoprecipitate with constitutively active RHO-1(G14V) (Fig. 2A), confirming that the interaction is conserved between mammals and nematodes. As a control, we also expressed human VSV-tagged DGK $\theta$, along with myctagged constitutively active RhoA(G14V), and showed that they coimmunoprecipitated (Fig. 2A).

To identify the region of DGK-1 required for coimmunoprecipitation with RHO-1, we expressed various deletions of DGK-1 (data not shown). We found that the last 247 amino acids of DGK-1 were sufficient for binding to constitutively active RHO-1(G14V) and, at a lower level, to wild-type RHO-1 (Fig. 2B). This DGK-1 fragment contains the DGK accessory domain, which is C-terminal to the DGK catalytic domain in all DAG kinases (Sakane and Kanoh 1997). The equivalent C-terminal fragment of human DGK $\theta$ was also able to interact with constitutively active human RhoA(G14V) with a higher affinity than with wild-type RhoA, and this interaction was partially blocked by expression of C3 transferase (Fig. 2C).

In our initial studies, we discovered that RhoA constructs from three different sources contained an additional undocumented mutation (F25N). This mutation significantly reduced the interaction between constitutively active RHO-1(G14V) and DGK-1 (Fig. 2B). Binding of the human RhoA (G14VF25N) to DGK $\theta$ was also significantly reduced by this amino acid substitution (Fig. 2C). We tested the effect of the F25N mutation on the ability of human myc-tagged RhoA(G14V) expressed in Cos 7 cells to bind other effectors. The F25N mutation did not alter the ability of constitutively active RhoA $(\mathrm{G} 14 \mathrm{~V})$ to bind GST fusions of other Rho effectors: PKN(1-511), mDia(47-257), and ROCKII(348-1018) (Fig. 2D). Thus, the F25N mutation selectively reduces Rho binding to DGK $\theta$, rather than all effectors. 
Figure 2. DGK-1 interacts with RHO-1. (A) Both full-length human DGK $\theta$ (VSV-tagged) and constitutively active RhoA(G14V) (myc-tagged) (left panels) or their C. elegans orthologs DGK-1 (HA-tagged) and RHO-1(G14V) (myc-tagged) (right panels) coimmunoprecipitated when coexpressed in Cos 7 cells. Cells were lysed and RHO-1 immunoprecipitated with the anti-myc monoclonal antibody 9E10. (Upper panel) Coimmunoprecipitated DGK $\theta$ or DGK-1 was visualized by Western blotting. Lower panels show expression controls (total lysates). The ratio of coimmunoprecipitated DGK $\theta$ (IP) to total DGK $\theta$ (VSVtotal) was determined by densitometry and is indicated below each lane. $(B)$ Cos7 cells were cotransfected with truncated DGK-1 (HA-DGK-1acc) and wild-type, G14V, or G14VF25N RHO-1 (myc-tagged). DGK-1 acc (lane 1) coimmunoprecipitated with RHO$1(\mathrm{G} 14 \mathrm{~V})$ with higher affinity than wild-type RHO-1 (lane 2), but this interaction was strongly reduced with the RHO-1(G14VF25N) mutant (lane 3). Lanes 4-6 show single-transfection controls. The ratio of coimmunoprecipitated DGK-1(IP) to total DGK1(HAtotal) was determined by densitometry and is indicated below each lane. $(C)$ Cos7 cells were cotransfected with truncated human DGK $\theta$ (VSV DGK $\theta$ acc), together with either wild-type, G14V or G14VF25N RhoA (myc-tagged), and C3 transferase. DGK $\theta$ acc coimmunoprecipitated with RhoA(G14V) (lane 1) with a higher affinity than wild-type RhoA (lane 2) or RhoA(G14VF25N) (lane 4). (Lane 3) Coexpression of $\mathrm{C} 3$ transferase partially decreased binding of wild-type RhoA to truncated DGK $\theta$. Lanes 5-6 show single-transfection controls. The ratio of coimmunoprecipitated DGK $\theta$ (IP) to total DGK $\theta$ (VSVtotal) was determined by densitometry and is indicated below each lane. $(D)$ G14V or G14VF25N RhoA (myc-tagged) were transfected into Cos7 cells. Cells were lysed, and lysates were incubated with GST fusions of PKN, mDia, or ROCKII. Following washing, bound RhoA was detected by Western blotting and quantified using densitometry. The data represent the mean of three separate experiments; error bars indicate the standard error of the mean. The additional F25N mutation does not block the binding of RhoA(G14V) to PKN, mDia, or ROCKII. No significant differences were detected between G14V and G14VF25N RhoA using a two-tailed $t$-test $(p>0.02)$.

The strength of RHO-1(G14V) binding to DGK-1 correlates with the potentiation of ACh release

To assess the effect of reduced RHO-1 binding to DGK-1 on neurotransmitter release, we expressed RHO1 (G14VF25N) presynaptically using the unc-17 promoter. Presynaptic expression of the poor DGK-1-binding RHO-1(G14VF25N) caused loopy locomotion, but the rate of locomotion, while higher than in wild-type animals, was slower than in animals with neuronal expression of constitutively active RHO-1(G14V), which binds more strongly to DGK-1 (Fig. 3A). In addition, presynaptic expression of RHO-1(G14VF25N) resulted in animals with an intermediate response to aldicarb treatment, between the response of animals expressing RHO$1(\mathrm{G} 14 \mathrm{~V})$, which retains full binding to DGK-1, and wildtype (Fig. 3B). If RHO-1(G14VF25N) is defective only because of its inability to bind and inhibit DGK-1, then removal of DGK-1 should compensate for the F $25 \mathrm{~N} \mathrm{mu-}$ tation. This was the case: The locomotion and aldicarb sensitivity of $d g k$-1-null animals expressing the RHO1(G14VF25N) mutant that bound DGK-1 poorly was indistinguishable from animals expressing RHO- $1(\mathrm{G} 14 \mathrm{~V})$ (Fig. 3A,B). If RHO-1 acts through inhibition of DGK-1, then we would predict that $d g k-1$ mutations should not enhance the locomotion of animals expressing RHO1(G14V). Animals expressing RHO-1(G14V) but lacking DGK-1 did not show significantly increased locomotion rates when compared with RHO-1(G14V) in a wild-type background (Fig. 3A), which suggests that RHO-1 and DGK-1 act in the same pathway.

\section{RHO-1 also stimulates ACh release through a DGK-1-independent pathway}

If RHO-1 stimulates ACh release exclusively by inhibiting DGK-1, then the removal of DGK-1, using a dgk-1null mutant, should completely suppress the effects of lowering endogenous RHO-1 signaling. The reduced locomotion and ACh release effects of C3 transferase expressed from the unc-17 promoter were suppressed in a dgk-1-null mutant (Fig. 4A,B), but not completely. Thus, even in the complete absence of DGK-1, the inhibition of endogenous RHO- 1 activity by C 3 transferase is still capable of reducing locomotion and $\mathrm{ACh}$ release. This suggests that RHO-1 activates two presynaptic pathways, one DGK-1-dependent and one DGK-1-independent, in stimulating C. elegans locomotion. 

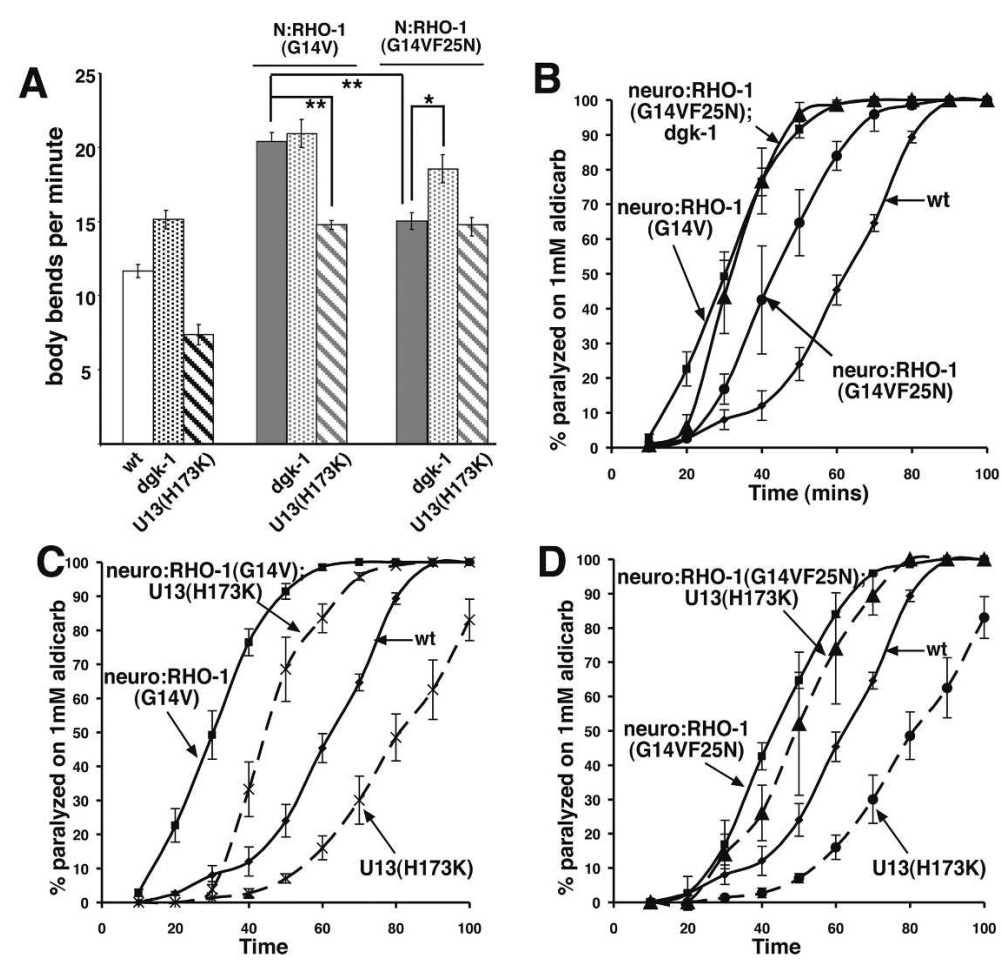

Figure 3. RHO-1 enhancement of acetylcholine release is partially dependent on binding to DGK-1 and binding of UNC-13 to DAG. (A) Body bends per minute were counted for wild-type, N:RHO-1(G14V), and N:RHO-1(G14VF25N) animals in wild-type, $d g k$ 1(sy428), and unc-13(s69);UNC13S(H173K)::GFP backgrounds. Presynaptic (unc-17 promoter) expression of the RHO-1(G14VF25N) mutant, which binds DGK-1 poorly, increases locomotion, but not as strongly as RHO-1(G14V). $d g k-1$ (sy428) increased locomotion of animals expressing the poor DGK-1binding RHO-1(G14VF25N) mutant but not RHO$1(\mathrm{G} 14 \mathrm{~V})$. The non-DAG-binding UNC-13 (H173K) mutant suppresses the locomotion of RHO-1(G14V) animals to a level equivalent to that of RHO1(G14VF25N) animals. dgk-1(sy428) animals expressing RHO-1(G14VF25N) moved slightly slower compared with RHO-1(G14V); $d g k-1$ (sy428), although this was not significant $(p>0.02)$. Error bars indicate standard error of the mean. Statistical analysis was performed using a two-tailed $t$-test and significance is indicated using one asterisk $\left(^{\star}\right) 0.001<p<0.02$, and two asterisks $\left({ }^{\star \star}\right) p<0.001$. $(B)$ Presynaptic expression of RHO-1(G14V), and to a lesser extent the poor DGK-1-binding RHO-1(G14VF25N), cause hypersensitivity to aldicarb, suggesting that binding to DGK-1 is required for the full effect of RHO-1 on ACh release. In a $d g k-1$ (sy428) mutant the weak DGK-1-

binding RHO-1(G14VF25N) shows the same hypersensitive response to aldicarb as the RHO-1(G14V) mutant. $(C, D)$ Replacement of endogenous UNC-13 with transgenic expression of a non-DAG-binding UNC-13S (H173K) mutant [unc-13(s69);nzIs25] partially reduced the aldicarb sensitivity resulting from the expression of RHO-1(G14V) expressed from the unc-17 promoter [neuro:RHO$1(\mathrm{G} 14 \mathrm{~V}) ; \mathrm{U} 13(\mathrm{H} 173 \mathrm{~K})](C)$, but not the aldicarb sensitivity resulting from unc-17-driven expression of the poor DGK-1-binding RHO$1(\mathrm{G} 14 \mathrm{VF} 25 \mathrm{~N})$ mutant [neuro:RHO-1(G14VF25N);U13(H173K)] (D). Error bars indicate standard error of the mean.

\section{RHO-1 increases the abundance of UNC-13 at sites of ACh release}

We have previously shown that DGK-1 acts within the motor neurons to inhibit ACh release by removal of DAG (Nurrish et al. 1999). DAG increases ACh release in the motor neurons by the recruitment of the DAGbinding protein UNC-13 to neurotransmitter release sites (Lackner et al. 1999; Nurrish et al. 1999). Thus, we expected that RHO-1-mediated inactivation of DGK-1 would increase DAG levels and alter UNC-13 localization. Consistent with this, expression of constitutively activated RHO-1(G14V) from the heat-shock promoter caused an increase in the number of UNC-13S::YFP punctate structures in the dorsal nerve cord, where neuromuscular junctions are located (Fig. 5A-D,G). To confirm that the relocalization of UNC-13 was due to changes in DAG levels, we expressed a mutant form of UNC-13, H173K, which is unable to bind to DAG (Kazanietz et al. 1995; Zhang et al. 1995; Betz et al. 1998). The non-DAG-binding UNC-13S(H173K)::GFP failed to change its localization in response to the expression of constitutively activate RHO-1(G14V) (Fig. 5E,G). Thus, RHO-1(G14V) regulates the localization of UNC$13 \mathrm{~S}$ in the dorsal nerve cord in a DAG-dependent manner.

To confirm that the UNC-13S::YFP-containing punctate structures were sites of neurotransmitter release, we carried out double-labeling experiments, using
SNB-1::CFP expressed from a motor neuron-specific promoter and UNC-13S::YFP expressed from its own pan-neural UNC-13S promoter. In the absence of RHO1 (G14V) expression, UNC-13S::YFP is diffusely localized within the motor neurons with a few discreet
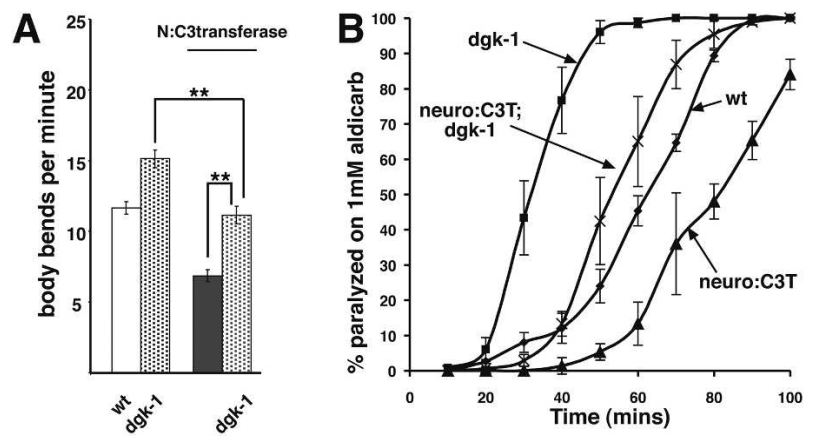

Figure 4. RHO-1 enhances acetylcholine release by DGK-1dependent and -independent mechanisms. Presynaptic expression (from the unc-17 promoter, N:C3 transferase or N:C3T) of C3 transferase reduces the locomotion rate $(A)$ and aldicarb sensitivity (B) of $d g k-1$ (sy428) mutant animals, although not to the same level as animals with presynaptic expression of C3 transferase in nonmutant animals, indicating that some but not all RHO-1 enhancement of ACh release occurs via inhibition of DGK-1. Error bars indicate standard error of the mean. Statistical analysis was performed using a two-tailed $t$-test, and two asterisks $\left(^{\star \star}\right)$ indicates significance $(p<0.001)$. 
McMullan et al.

Figure 5. RHO-1(G14V) regulates the distribution of UNC-13S::YFP in the dorsal nerve cord. In $A-E$, digital images were converted from gray scale into a 32-color lookup table (NIH Image) to visualize pixel intensities. $(A-D)$ UNC-13S::YFP (expressed from the internal unc-13S promoter) was primarily diffusely distributed in the dorsal nerve cord axons of untreated $(A)$ and heatshocked $(B)$ wild-type animals, as well as non-heat-shocked animals containing the hs::RHO-1(G14V) array (hs::RHO-1*) (C). $(D)$ UNC-13S::YFP became more punctate in the dorsal cords of heat-shocked hs::RHO-1(G14V) (hs::RHO-1*) animals. (E) Unlike the wild-type protein, UNC-13S $(\mathrm{H} 173 \mathrm{~K}):: \mathrm{GFP}$, which is predicted not to bind to DAG (Betz et al. 1998), remains diffusely distributed in heat-shocked hs::RHO-1(G14V) (hs::RHO-1*) animals. (F) UNC-13S is localized to acetylcholine release sites in heat-shocked hs::RHO$1(\mathrm{G} 14 \mathrm{~V})$ animals. Motor neuron nerve terminals and UNC-13S were simultaneously visualized in heat-shocked hs::RHO$1(\mathrm{G} 14 \mathrm{~V})$ animals with CFP-tagged synaptobrevin (top) and YFP-tagged UNC-13S (middle). (Bottom) In the merged image, it is clear that most SNB-1::CFP puncta are colocalized with a UNC-13S::YFP punctum. The SNB-1::CFP construct was specifically expressed in motor neurons (with the acr-2 promoter). The UNC-13S::YFP construct was expressed throughout the nervous system with the unc-13S promoter. $(G)$ Numbers of either UNC$13 S::$ YFP or non-DAG-binding UNC13S(H173K)::GFP puncta in dorsal nerve cords were compared for animals carrying the hs:RHO-1(G14V), or nontransgenic (w.t.) animals plus or minus heat shock, with the indicated UNC-13 marker. Digital images were thresholded according to pixel intensity, and puncta were counted manually. Bars are means $\pm \mathrm{SEM}$; numbers of animals counted are given in brackets. Significance was determined by a two-tailed $t$-test. $\left(^{*}\right)$ Significantly differs from nonheat-shocked hs::RHO-1(G14V) $(p<0.02)$.
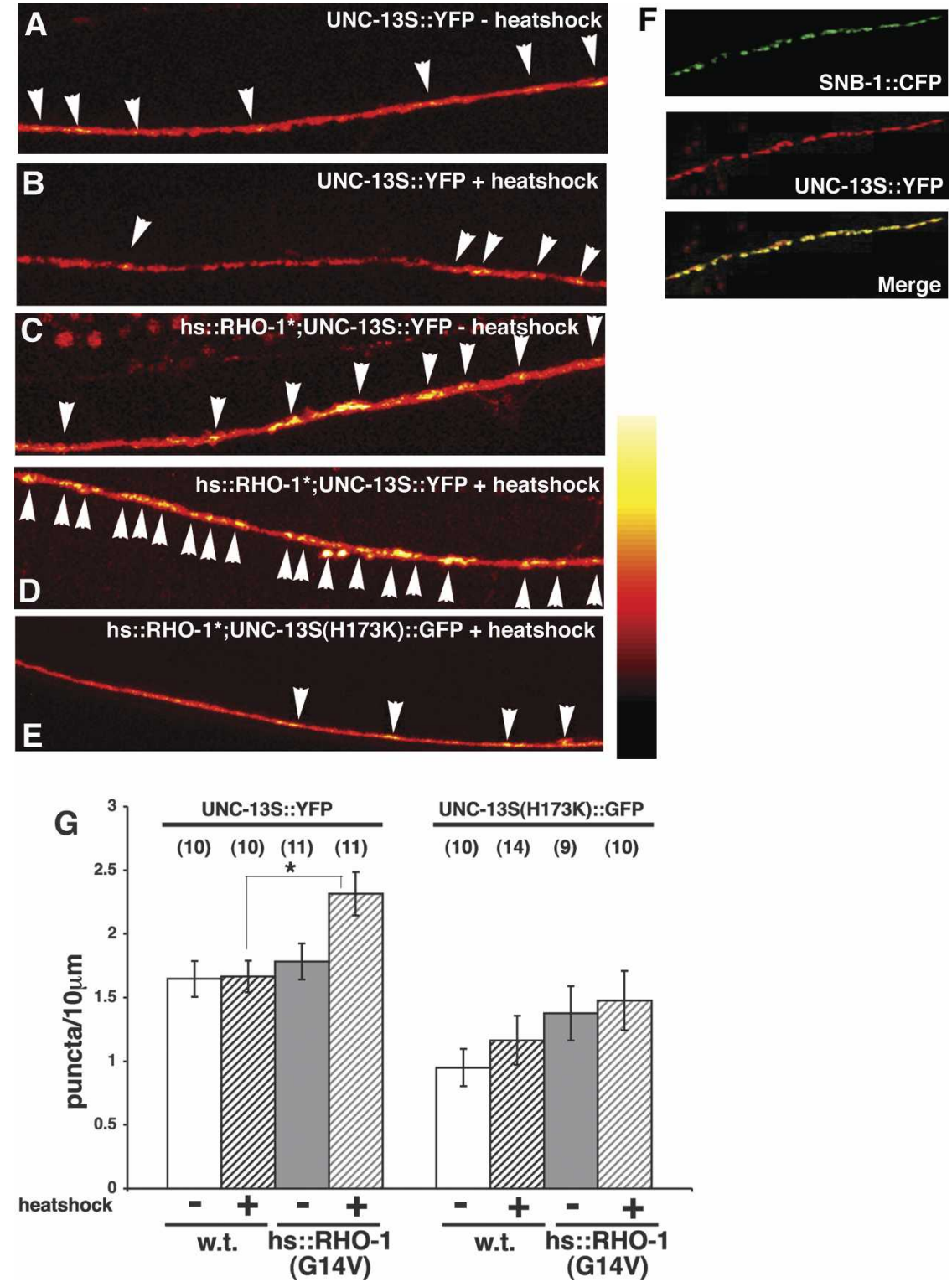

puncta that are colocalized with SNB-1::CFP puncta (data not shown). Upon heat-shock-induced expression of RHO-1(G14V), the UNC-13S::YFP became more punctate and colocalized with the SNB-1::CFP puncta (Fig. 5F), although the UNC-13S::YFP appeared to spread beyond the sites defined by SNB-1::CFP. Although the number of UNC-13S::YFP puncta was increased in animals expressing RHO-1(G14V), the number of SNB$1::$ CFP puncta was unaffected (Supplementary Fig. 2), demonstrating that $\mathrm{RHO}-1(\mathrm{G} 14 \mathrm{~V})$ recruits UNC-13S to pre-existing synapses. These results indicate that RHO-1 causes a spatially restricted increase in DAG levels at sites of neurotransmitter release.
DAG-binding mutants of UNC-13 partially block the RHO-1(G14V)-induced increase in neurotransmitter release

In both $C$. elegans and mice hippocampal neurons, stimulation of neurotransmitter release in response to the DAG analog phorbol ester is substantially blocked by the UNC-13 (H173K) mutation, which is unable to bind DAG (Lackner et al. 1999; Rhee et al. 2002). Expression of RHO-1 $(\mathrm{G} 14 \mathrm{~V})$ in animals possessing only the nonDAG-binding UNC-13 H173K mutant results in a partial block of RHO-1(G14V)'s effects, reducing both rates of locomotion and sensitivity to aldicarb (Fig. 3A,C). How- 
ever, the constitutively active $\mathrm{RHO}-1(\mathrm{G} 14 \mathrm{~V})$ retains the ability to strongly increase $\mathrm{ACh}$ release in UNC13(H173K) animals, suggesting that RHO-1 stimulates release by both UNC-13-dependent and UNC-13-independent mechanisms. The UNC-13(H173K) mutant has very little effect on the aldicarb-induced paralysis of animals expressing the RHO-1(G14VF25N) mutant, which binds DGK-1 poorly (Fig. 3A,D). These results suggest a model in which RHO-1 acts presynaptically to increase ACh release via two pathways-one depending on the inhibition of DGK-1, which causes an increase in DAG and relocalization of UNC-13 to release sites, and a second that is independent of both DGK-1 and the ability of UNC-13 to bind to DAG.

\section{Acute regulation of $\mathrm{RHO}-1$ increases muscle sensitivity to acetylcholine}

The heat-shock promoter we used to acutely express constitutively active RHO-1(G14V) or C3 transferase drives expression in more cell types than just neurons. Thus, we investigated whether changes in RHO-1 signaling could also affect muscle responsiveness to ACh. To do this we tested for sensitivity to levamisole, an activator of the muscle nicotinic ACh receptors. We found that heat-shock-driven expression of RHO-1(G14V) caused animals to become hypersensitive to levamisole (Fig. 6A), suggesting that RHO-1 may also increase synaptic strength at a site distinct from the motor neurons. Surprisingly, expression of C3 transferase from both the heat-shock and unc-17 promoter caused a slight increase in muscle sensitivity to levamisole (Fig. 6B,C). Presynaptic expression of either the constitutively active RHO1 (G14V) or the poor DGK-1-binding RHO-1(G14VF25N) from the unc-17 promoter caused no change in the response to levamisole (Fig. 6C).

\section{Discussion}

We show that inhibition of endogenous RHO-1 activity with C3 transferase causes a dramatic switch in animal behavior toward slow locomotion and decreased amplitude of body bends, suggesting that signaling through RHO-1 is required for wild-type levels of locomotion. Coincident with these behavioral changes, C3 transferase decreases release of $\mathrm{ACh}$ when expressed either acutely in various cell types, including motor neurons and muscles (heat-shock expression in adults), or in ACh-releasing neurons (from the unc-17 promoter). Transgenic expression of a constitutively active RHO1(G14V) mutant has the opposite effect: Animals move more quickly and with exaggerated body bends. Markers for both gross motor neuron morphology and for ACh release sites reveal no changes in response to increased RHO-1 signaling. Thus, RHO-1 acts presynaptically at pre-existing synapses to stimulate neurotransmitter release at the C. elegans neuromuscular junction and thereby modulates locomotion.

The hyperactive locomotion behavior and increased aldicarb sensitivity of RHO-1(G14V)-expressing animals is very similar to that of both wild-type animals exposed to the DAG analog phorbol ester and animals with lossof-function mutations in goa-1 or $d g k-1$ (Mendel et al. 1995; Segalat et al. 1995; Hajdu-Cronin et al. 1999; Lackner et al. 1999; Miller et al. 1999; Nurrish et al. 1999|. In the case of goa-1 mutants, hyperactive locomotion is caused by an increase in both DAG and the DAG-binding UNC-13 protein at ACh release sites (Nurrish et al. 1999). Acute expression of RHO-1(G14V) also promotes DAG-dependent accumulation of UNC-13 at sites of ACh release in dorsal motor neurons. UNC-13 is a core component of the synaptic release machinery essential for the priming of synaptic vesicle exocytosis (Richmond
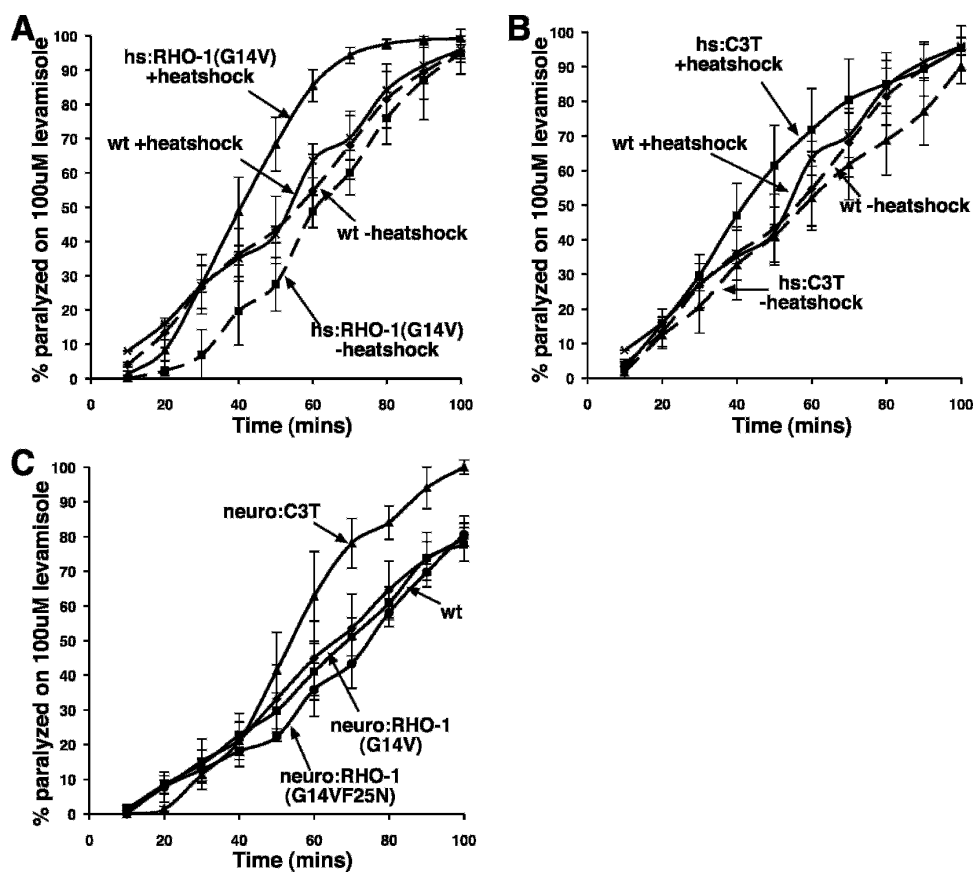

Figure 6. Acute expression of RHO-1(G14V) increases muscle response to acetylcholine (ACh). Muscle response to ACh was measured by determining the onset of paralysis induced by the nicotinic agonist levamisole at $100 \mu \mathrm{M}$. (A) Heat-shock expression of a constitutively active RHO- $1(\mathrm{G} 14 \mathrm{~V})$ mutant [hs:RHO$1(\mathrm{G} 14 \mathrm{~V})+$ heat shock] resulted in a faster onset of paralysis in the presence of levamisole compared with non-heat-shocked [hs:RHO-1(G14V) - heat shock] and nontransgenic controls (wt - heat shock and wt + heat shock). (B) Heat-shock expression of the RhoA inhibitor C3 transferase (hs:C3T + heat shock) caused a very slight increase in onset of paralysis in response to levamisole compared with non-heat-shocked (hs:C3T heat shock) and nontransgenic controls (wt-heat shock and $\mathrm{wt}+$ heat shock). (C) Animals with presynaptic (p.unc-17) expression of RHO-1(G14V) [neuro:RHO$1(\mathrm{G} 14 \mathrm{~V})]$ or RHO-1(G14VF25N) [neuro:RHO$1(\mathrm{G} 14 \mathrm{VF} 25 \mathrm{~N})]$ paralyzed at the same rate as nontransgenic $(w t)$ animals when exposed to levamisole. Presynaptic expression of C3 transferase from the unc-17 promoter (neuro:C3T) causes a slight hypersensitivity of the muscles to ACh, as shown by a slight increase in response to levamisole. Error bars indicate standard error of the mean. 
et al. 1999, 2001). Thus, we are able to show that RHO-1 signaling modulates a core component of the synaptic release machinery.

How does RHO-1(G14V) increase DAG at release sites? DGK- 1 and DGK $\theta$ are DAG kinases, a family of lipid kinases that phosphorylate the membrane-bound second messenger DAG to phosphatidic acid (for reviews, see van Blitterswijk and Houssa 2000; Luo et al. 2004). As noted above, animals expressing constitutively active RHO-1(G14V) have a similar phenotype to $d g k-1$ mutants, and $d g k-1$ mutants do not increase the locomotion of animals expressing RHO- $(\mathrm{G} 14 \mathrm{~V})$ in the motor neurons, suggesting they act in the same pathway. Human RhoA has been shown to inhibit DGK $\theta$ in vitro, suggesting a role for RhoA in regulating DAG levels (Houssa et al. 1999), and now we show that C. elegans RHO-1 is able to bind to DGK-1. Furthermore, a RHO-1 mutant with a reduced ability to bind DGK-1, RHO1(G14VF25N), has a reduced ability to stimulate ACh release. Our results are most consistent with a signal transduction cascade leading from activation of RHO-1, through an increase in DAG, via inhibition of DGK-1, to the recruitment of UNC-13 to sites of ACh release. We and others (Hajdu-Cronin et al. 1999; Lackner et al. 1999; Miller et al. 1999; Nurrish et al. 1999) have also shown that two antagonistic G-protein signaling pathways control DAG levels in C. elegans motor neurons. Thus, we can place this novel RHO-1 signaling pathway within a larger model of how DAG levels, and thus neurotransmitter release, are regulated within C. elegans motor neurons (Fig. 7).

In $d g k$-1-null mutants, UNC-13 relocates to the motor neuron plasma membrane, rather than to specific release sites (Nurrish et al. 1999). In contrast, RHO-1(G14V) causes DAG levels to increase only at release sites, as shown by the specific localization of DAG-binding UNC-13 (although the UNC-13 does spread further than the SNB-1 release-site marker). This localized increase in DAG suggests that activation of RHO-1 does not cause a generalized inhibition of DGK-1, but rather a specific inhibition of DGK-1 at sites of neurotransmitter release, thus ensuring that DAG levels increase only at sites of ACh release.

Two lines of evidence indicate that RHO-1 can enhance ACh release independently of DGK-1 inhibition. First, inhibition of endogenous RHO-1 by C3 transferase still reduces locomotion and $\mathrm{ACh}$ release in a dgk-1-null mutant, inconsistent with a model in which RHO-1 signals exclusively by inhibiting DGK-1. Second, the nonDAG-binding UNC-13(H173K) mutant only partially blocks signaling from the constitutively active RHO1(G14V). The UNC-13(H173K) mutant almost completely blocks the ability of low concentrations of the DAG analog PMA to stimulate release, demonstrating that UNC-13 is the primary high-affinity target of DAG effects on ACh release (Lackner et al. 1999). These results suggest that RHO-1 can stimulate ACh release by a second, DGK-1-and DAG-independent, pathway. According to this model, we would predict that expression of RHO-1(G14V), which activates both pathways, would

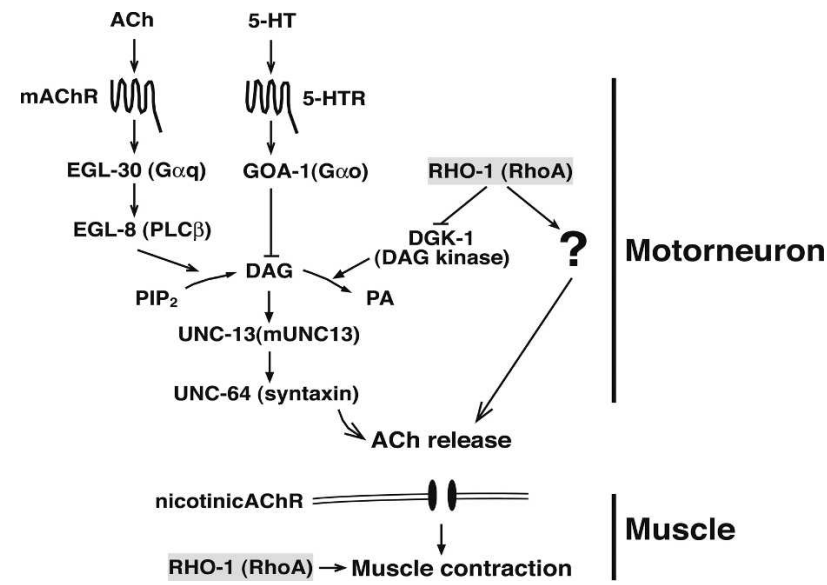

Figure 7. RHO-1 acts via three distinct pathways to increase synaptic strength at the C. elegans neuromuscular junction. We propose an addition to the competing G-protein pathway model in C. elegans motor neurons (Lackner et al. 1999; Miller et al. 1999; Nurrish et al. 1999). To this pathway we added RHO-1 as a negative regulator of DGK-1. Activation of RHO-1 causes a loss of DGK-1 activity, leading to a stabilization of diacylglycerol (DAG) levels at sites of release. Presynaptic DAG recruits UNC-13 to release sites, and the accumulation of UNC-13 at release sites correlates with increases in ACh release. We further propose that RHO- 1 acts by a second presynaptic pathway that is independent of DGK-1 inhibition and of DAG-binding to UNC-13. Finally, our data suggest that RHO-1 expression at a site other than the motor neuron potentiates the response of the muscle to ACh.

further increase $\mathrm{ACh}$ release and locomotion rates in $d g k-1$ mutants, which mimics activation of only one of the RHO-1 pathways. However, we did not observe any such increase; possibly the locomotion rates of $d g k-1$ animals may represent the maximum rate in our assay that cannot be increased, even by activation of the DGK1-independent pathway.

Interestingly, $\mathrm{ACh}$ release stimulated by expression of the RHO-1(G14VF25N) mutant, which binds to DGK-1 poorly, is not inhibited by the UNC-13(H173K) mutant. Perhaps RHO-1(G14VF25N) fails to inhibit DGK-1 and stimulates ACh release entirely by the DGK-1-independent pathway. Accordingly, null mutations in $d g k-1$ enhance both the rate of locomotion and aldicarb sensitivity of animals expressing the poor DGK-1-binding RHO1 (G14VF25N) to equivalent levels of the strong DGK-1binding RHO-1(G14V). It appears that RhoA constructs containing the $\mathrm{F} 25 \mathrm{~N}$ mutation are quite common, and indeed we were unable to locate a source of RhoA without this mutation. The F25 residue is immediately $\mathrm{N}$ terminal to the Rho switch 1 region and makes contacts with the Rho-binding domain in a RhoA-PKN crystal structure (Maesaki et al. 1999). It is possible that effects of RhoA on DAG signaling have been previously missed due to the use of the RhoA(F25N) mutant.

What is the DGK-1-independent pathway by which RHO-1 stimulates release presynaptically? One possibility is that RHO-1 reorganizes presynaptic actin (Hall 1998; Dillon and Goda 2005). Changes in the actin cyto- 
skeleton are a prerequisite for exocytosis, enabling docking and fusion of secretory vesicles with the plasma membrane (for review, see Dillon and Goda 2005). In neurons, changes in presynaptic actin modulate vesicle fusion at active zones (Morales et al. 2000), increase the movement of actin-tethered synaptic vesicles from the reserve pool to active zones (Mochida et al. 1994; Kuromi and Kidokoro 1998; Hilfiker et al. 2005), and promote endocytosis of synaptic vesicles following neurotransmitter release (Shupliakov et al. 2002). Alternatively, the DGK-1-independent RHO-1 pathway could involve the aggregation of synaptic proteins to existing synapses (Wang et al. 2005). Constitutively active RHO-1(G14V) does not generate new aggregations of synaptobrevin (SNB-1)-labeled synaptic vesicles, ruling out the formation of new functional synapses. However, we were unable to measure numbers of SNB-1 agregations in C3 transferase-expressing animals, so it is possible that reducing RHO-1 activity could cause the removal of release sites. A genetic screen in C. elegans for suppressors of the increased $\mathrm{ACh}$ release phenotype of neuronally expressed RHO-1(G14V) (E. Hiley and S.J. Nurrish, unpubl.) has identified a number of recessive mutants; the characterization of these mutants should shed light on the DGK-1-independent RHO-1 signaling pathway.

A third mechanism by which RHO-1 enhances synaptic strength is revealed by the finding that expression of RHO-1(G14V) from the widely expressed heat-shock promoter causes hypersensitivity of the muscles to ACh. The simplest explanation for this finding is that the constitutively active RHO-1(G14V) acts post-synaptically to increase muscle responsiveness to ACh. Rho is known to increase the contraction by actin-myosin filaments via Rho-associated kinase, which phosphorylates myosin light chain (Kimura et al. 1996). Alternatively, RHO-1 stimulation could increase clustering (Weston et al. 2003) or activity of the nicotinic ACh receptors by an as yet unidentified mechanism. Unexpectedly, we find that heat-shock and neuronal expression of $\mathrm{C} 3$ transferase also causes muscles to become slightly hypersensitive to ACh. The decreased release of ACh triggered by the C3 transferase-dependent inhibition of RHO-1 may induce homeostatic compensation in the muscles. A similar compensation effect has been observed at the Drosophila neuromuscular junction (Davis and Goodman 1998).

All of the components in our proposed DGK-1-dependent Rho signaling pathway are conserved in mammals, including RHO-1(RhoA), DGK-1(DGK $\theta)$, and UNC-13 (munc13-1). Since RhoA, DGK $\theta$, and munc13-1 are present in the mammalian brain, it is possible that RhoA regulates neurotransmitter release in mammals by modulating DAG levels. If so, DAG kinases and their regulators might be good targets for drugs to alter synaptic signaling in the brain.

\section{Materials and methods}

\section{Strains}

The N2 (wild-type) strain was obtained from the Caenorhabditis Genetics Center (University of Minnesota). The dgk- 1(sy428) (a gift of P. Sternberg, California Institute of Technology, Los Angeles, CA) is an early nonsense mutation that produces no detectable DGK-1 protein (Jose and Koelle 2005), and thus likely represents a null mutant. For locomotion and aldicarb assays, UNC-13 constructs were expressed in unc-13(s69) mutants. All strains were cultivated at $20^{\circ} \mathrm{C}$ unless otherwise stated and were maintained as described previously (Brenner 1974).

\section{Transgenes and germline transformation}

Plasmids (listed as QT\#, SJN, or RJM) were constructed by standard techniques, and sequences were verified by sequencing. Transgenic strains (listed as QT) were isolated by microinjection of $100 \mathrm{ng} / \mathrm{\mu L}$ of plasmid, unless otherwise described below, together with $t t x-3:: g f p$ at $100 \mathrm{ng} / \mu \mathrm{L}$ (a gift of O. Hobert, Columbia University, New York, NY) or unc-122::gfp at $50 \mathrm{ng} / \mathrm{\mu L}$ (a gift of P. Sengupta, Brandeis University, Waltham, MA) as a marker. Unless otherwise stated, all injections were performed into N2 animals. Plasmids and transgenic strains were constructed as follows.

RHO-1 transgenes cDNA for RHO- 1 was isolated from whole C. elegans RNA using the SMART RACE kit (Clontech) as per the manufacturer's instructions. The sequence was modified by PCR to create the G14V mutation and subcloned either into the pPD79.48 (a gift of A. Fire, Stanford University, Stanford, CA) heat-shock vector (QT\#42) or a vector driving expression from the unc-17 cholinergic-specific promoter (a 3213-base-pair [bp] fragment located -1226 to -4439 bp 5' of the unc-17 start codon, a gift of J. Kaplan, Massachusetts General Hospital, Boston, MA) (QT\#220). These plasmids were injected at $5 \mathrm{ng} / \mu \mathrm{L}$. nzIs1 and nzIs28 contain an integrated version of the QT\#42 and QT\#220 plasmids, respectively. Plasmids SJN349 and SJN327 are identical to QT\#42 and QT\#220, respectively, except they contain a point mutation resulting in the rho-1 amino acid change F25N. Stable arrays of SJN349 (nzEx99) and SJN327 (nzEx72) were generated by injection into wild-type animals. Transgenes expressing RHO-1 N-terminally tagged with GFP, MYC, or HA lost their ability to stimulate ACh release and locomotion.

C3 transferase transgenes The C3 transferase coding sequence was a gift of R. Treisman (Cancer Research UK, London, UK). C3 transferase was $\mathrm{N}$-terminally tagged with GFP (derived from pPD95.75, a gift from A. Fire) and expressed from a heat-shock promoter (QT\#99) or from the unc-17 promoter (SJN266). These transgenes were injected at $1 \mathrm{ng} / \mu \mathrm{L}$. $n z E x 4$ and $n z E x 95$ contain extrachromosomal versions of QT/99 and SJN266, respectively.

UNC-13 transgenes For UNC-13 and SNB-1 visualization: nuIs59 contains rol-6, KP\#291 (which encodes a full-length unc$13 S:$ YFP fusion gene that restores locomotion to animals carrying unc-13 alleles) and KP\#282 (which encodes a SNB-1::CFP fusion expressed from the acr-2 promoter [a gift of Y. Jin, University of California at Santa Cruz, Santa Cruz, CA], which is expressed in the neurons VA, DA, VB, DB, IL1, RMD, and PVQ) integrated in a wild-type background. KP\#273 encodes UNC$13 S:$ GFP, which contains a point mutation resulting in the amino acid change H173K. nzIs25 contains an integrated version of KP\#273 in an unc-13 (s69) background. Strain QT262 has both the nzIs1[hs::rho-1(G14V)] and nuIs59 integrants. Strain QT426 has both the nuIs59 and nzIs28[N::rho-1(G14V)] integrants. Strain QT336 has the nzIs1 and nzIs25 integrated arrays in the unc-13(s69) background. As rol-6 expression interferes with locomotion and aldicarb assays, we used nuIs46, which is an integration of the KP\#268 (UNC-13S::GFP) plasmid instead 
of nuIs59. Strain QT148 has both the nzIs1 and nuIs46 integrants in the unc-13(s69) background. Strain QT328 has the nzIs25 and nzIs28 integrated arrays in an unc-13(s69) background. nzEx122 has SJN327 [p.unc-17::RHO-1(G14VF25N)],

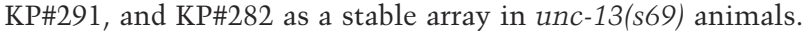

\section{Induction of heat-shock-inducible transgenes}

In all cases, expression from the heat-shock promoter was achieved using two rounds of heat shock for $60 \mathrm{~min}$ at $30^{\circ} \mathrm{C}$, separated by $30 \mathrm{~min}$ at $20^{\circ} \mathrm{C}$, followed by recovery for $30 \mathrm{~min}$ at $20^{\circ} \mathrm{C}$.

\section{Microscopy}

Strains expressing punc-47:GFP, punc13s:UNC13S::YFP, pacr2:SNB-1::CFP, or punc-13s:UNC13S(H173K)::GFP were imaged by mounting on agarose pads and viewed on a Bio-Rad 2100 upright multiphoton confocal microscope with a Nikon $60 \times$ objective. Images were obtained using LaserSharp software (Bio-Rad). Digital images were processed to give maximum intensity projections of a $z$-series using Image (NIH). Images were blinded with respect to genotype and treatment, and UNC-13S::YFP and SNB-1::CFP puncta were counted manually. The following strains were used for microscopy: EG1285 [oxIs12], QT195 [nzIs1;oxIs12], QT316 [nzIs28;oxIs12], QT427 [nzEx95;oxIs12], KP1683 [nuIs59], QT262 [nzIs1;nuIs59], QT181 [nzIs25], QT336 [nzIs1;nzIs25], QT426 [nzIs28;nuIs59].

\section{Analysis of locomotion and sensitivity to drug treatment}

Sensitivity to $1 \mathrm{mM}$ aldicarb (Greyhound Chromatography) or $100 \mu \mathrm{M}$ levamisole (Sigma) on NGM plates with food was determined by analyzing the onset of paralysis as described previously (Nurrish et al. 1999). For each experiment, 25 animals were tested, and each experiment was repeated at least five times. For locomotion rates, the number of body bends per minute was counted by visual inspection, and animals were assayed on NGM plates in the presence of food. Each animal was assayed three times for $1 \mathrm{~min}$ each. At least five animals were tested in all cases.

\section{Plasmids for mammalian expression and recombinant protein purification}

pcDNA1DGK $\theta$ VSV was kindly provided by W.J. van Blitterswijk (Nederlands Kanker Instituut, Amsterdam, Netherlands). pcDNA3.1DGK $\theta$-accVSV containing residues 731-942 and including the accessory domain of DGK $\theta$ was generated by PCR. Plasmids for the expression of mammalian RhoA in the pRK5 vector were a gift from A. Hall (MRC Laboratory for Molecular Cell Biology, London, UK). These plasmids contained a phenylalanine to aspartate mutation at position 25 that was corrected by PCR (QT\#240). pEFC3 for expression of C3 in mammalian cells and pGexKG-PKN(1-511), pGexKG-mDia(47-257), and pGexKG-ROCKII(348-1018) for purification of recombinant proteins were gifts from R. Treisman. C. elegans rho-1 was amplified from C. elegans cDNA using the SMART RACE cDNA kit (Clontech). The rho-1 cDNA was cloned into pRK5 and Nterminally tagged with the MYC epitope (SJN361). This was further mutated by PCR to give the amino acid changes G14V (SJN307) and G14VF25N (SJN342). The $d g k-1$ cDNA (Nurrish et al. 1999) was cloned into the pCDNA3.1 mammalian expression vector and C-terminally tagged with the HA epitope (RJM001). The $d g k-1$ accessory domain, residues 703-950, was cloned into pCNA3.1 and C-terminally tagged with HA (SJN345).

\section{Cell culture and transfection}

Cos 7 cells were grown at $37^{\circ} \mathrm{C}$ in Dulbecco's modified Eagles medium supplemented with $10 \%$ fetal calf serum and antibiotics. Cell transfections were performed using Genejuice (Novagen) according to the manufacturer's instructions. In all cases, between 2 and $5 \mu \mathrm{g}$ of DNA was transfected, and cells were used for experiments $48 \mathrm{~h}$ after transfection.

\section{Immunoprecipitation and Western blotting}

Following transfection, cells were scrape lysed in $150 \mathrm{mM}$ $\mathrm{NaCl}, 50 \mathrm{mM}$ Tris- $\mathrm{HCl}(\mathrm{pH} 8.0), 5 \mathrm{mM} \mathrm{MgCl}, 1 \%$ Nonidet $\mathrm{P}-40$, and protease inhibitors. Immunoprecipitations were carried out with $\sim 200 \mu \mathrm{g}$ of cell lysate and $2 \mu \mathrm{g}$ of antibody using standard methods. Washes were performed in $150 \mathrm{mM} \mathrm{NaCl}, 50$ $\mathrm{mM}$ Tris- $\mathrm{HCl}(\mathrm{pH} 8.0), 5 \mathrm{mM} \mathrm{MgCl}{ }_{2}, 1 \%$ Nonidet P-40, and protease inhibitors. Immunoprecipitated proteins were subjected to SDS-polyacrylamide gel electrophoresis and immunoblotting, together with $20 \mu \mathrm{g}$ of total lysates, using standard methods. The following antibodies were used for immunoprecipitation and immunoblotting: anti-myc tag 9E10 (Santa Cruz), anti-VSV tag (Abcam), anti- $\beta$ tubulin E7 (Developmental Studies Hybridoma Bank, University of Iowa), and anti-HA (Santa Cruz). Densitometric analysis was performed using Quantity One software (Bio-Rad). We attempted to measure the DAG kinase activity of DGK-1 bound to RHO-1; however, levels of coimmunopreciptated DGK-1 were too low for results from the DAG kinase assay (Nurrish et al. 1999; Jose and Koelle 2005) to be reliable.

\section{GST pulldowns}

GST pulldowns using recombinant GST-PKN(1-511), GST$\mathrm{mDia}(47-257)$, and GSTROCKII(348-1018) were performed as described (Sahai et al. 1998) with the following modifications. pcDNA3.1mycV14RhoA and pcDNA3.1mycV14N25RhoA were transfected into Cos7 cells as described above. Forty-eight hours after transfection, cells were lysed in $150 \mathrm{mM} \mathrm{NaCl}, 50$ $\mathrm{mM}$ Tris- $\mathrm{HCl}(\mathrm{pH} 8.0), 5 \mathrm{mM} \mathrm{MgCl} 2,1 \%$ Nonidet P-40, and protease inhibitors. Two-hundred micrograms of cell lysate was incubated with $20 \mu \mathrm{g}$ of GST fusion protein for $2 \mathrm{~h}$ at $4^{\circ} \mathrm{C}$, and washes were performed. Following SDS-polyacrylamide gel electrophoresis and immunoblotting, bands were detected using a FluorSmax fluorimeter, and densitometric analysis was carried out using Quantity One software (Bio-Rad). Values represent the means of three separate experiments.

\section{Statistical analysis}

In all cases statistical analysis was performed using an unpaired two-tailed $t$-test. $P$ values between 0.02 and 0.001 (significant) are indicated on figures using one asterisk, and $P$ values of 0.001 or less (highly significant) are indicated with two asterisks.

\section{Acknowledgments}

We thank the following individuals for strains, plasmids, and advice: A. Hall, J. Kaplan, R. Treisman, W.J. van Blitterswijk, P. Sengupta, P. Sternberg, Y. Jin, A. Fire, J. Pitcher, and the Caenorhabditis Genetics Center. We thank A. Hall, M. Raff, Y. Goda, and M. Laycock for comments on the manuscript. E.H. is funded by a 4-year MRC Ph.D. studentship. 


\section{References}

Aktories, K. and Hall, A. 1989. Botulinum ADP-ribosyltransferase C3: A new tool to study low molecular weight GTPbinding proteins. Trends Pharmacol. Sci. 10: 415-418.

Alfonso, A., Grundahl, K., Duerr, J.S., Han, H.P., and Rand, J.B. 1993. The Caenorhabditis elegans unc-17 gene: A putative vesicular acetylcholine transporter. Science 261: 617-619.

Betz, A., Ashery, U., Rickmann, M., Augustin, I., Neher, E., Sudhof, T.C., Rettig, J., and Brose, N. 1998. Munc13-1 is a presynaptic phorbol ester receptor that enhances neurotransmitter release. Neuron 21: 123-136.

Brenner, S. 1974. The genetics of Caenorhabditis elegans. Genetics 77: 71-94.

Brose, N. and Rosenmund, C. 2002. Move over protein kinase C, you've got company: Alternative cellular effectors of diacylglycerol and phorbol esters. J. Cell Sci. 115: 4399-4411.

Brose, N., Betz, A., and Wegmeyer, H. 2004. Divergent and convergent signaling by the diacylglycerol second messenger pathway in mammals. Curr. Opin. Neurobiol. 14: 328-340.

Brown, A.M., O'Sullivan, A.J., and Gomperts, B.D. 1998. Induction of exocytosis from permeabilized mast cells by the guanosine triphosphatases Rac and Cdc42. Mol. Biol. Cell 9: $1053-1063$.

Chechlacz, M. and Gleeson, J.G. 2003. Is mental retardation a defect of synapse structure and function? Pediatr. Neurol. 29: $11-17$.

Chen, W. and Lim, L. 1994. The Caenorhabditis elegans small GTP-binding protein RhoA is enriched in the nerve ring and sensory neurons during larval development. J. Biol. Chem. 269: 32394-32404.

Dalpe, G., Zhang, L.W., Zheng, H., and Culotti, J.G. 2004. Conversion of cell movement responses to Semaphorin-1 and Plexin-1 from attraction to repulsion by lowered levels of specific RAC GTPases in C. elegans. Development 131: 2073-2088.

Dash, P.K., Orsi, S.A., Moody, M., and Moore, A.N. 2004. A role for hippocampal Rho-ROCK pathway in long-term spatial memory. Biochem. Biophys. Res. Commun. 322: 893-898.

Davis, G.W. and Goodman, C.S. 1998. Synapse-specific control of synaptic efficacy at the terminals of a single neuron. $\mathrm{Na}$ ture 392: 82-86.

Dillon, C. and Goda, Y. 2005. The actin cytoskeleton: Integrating form and function at the synapse. Annu. Rev. Neurosci. 28: $25-55$.

Etienne-Manneville, S. and Hall, A. 2002. Rho GTPases in cell biology. Nature 420: 629-635.

Ghijsen, W.E. and Miriam Leenders, A.G. 2005. Differential signaling in presynaptic neurotransmitter release. Cell. Mol. Life Sci. 62: 937-954.

Govek, E.E., Newey, S.E., and Van Aelst, L. 2005. The role of the Rho GTPases in neuronal development. Genes. \& Dev. 19: $1-49$.

Hajdu-Cronin, Y.M., Chen, W.J., Patikoglou, G., Koelle, M.R., and Sternberg, P.W. 1999. Antagonism between G(o) $\alpha$ and G(q) $\alpha$ in Caenorhabditis elegans: The RGS protein EAT-16 is necessary for $\mathrm{G}(\mathrm{o}) \alpha$ signaling and regulates $\mathrm{G}(\mathrm{q}) \alpha$ activity. Genes \& Dev. 13: 1780-1793.

Hall, A. 1998. Rho GTPases and the actin cytoskeleton. Science 279: 509-514.

Hilfiker, S., Benfenati, F., Doussau, F., Nairn, A.C., Czernik, A.J., Augustine, G.J., and Greengard, P. 2005. Structural domains involved in the regulation of transmitter release by synapsins. J. Neurosci. 25: 2658-2669.

Houssa, B., de Widt, J., Kranenburg, O., Moolenaar, W.H., and van Blitterswijk, W.J. 1999. Diacylglycerol kinase $\theta$ binds to and is negatively regulated by active RhoA. J. Biol. Chem. 274: 6820-6822.

Humeau, Y., Popoff, M.R., Kojima, H., Doussau, F., and Poulain, B. 2002. Rac GTPase plays an essential role in exocytosis by controlling the fusion competence of release sites. J. Neurosci. 22: 7968-7981.

Ishida, H., Zhang, X., Erickson, K., and Ray, P. 2004. Botulinum toxin type A targets RhoB to inhibit lysophosphatidic acidstimulated actin reorganization and acetylcholine release in nerve growth factor-treated PC12 cells. J. Pharmacol. Exp. Ther. 310: 881-889.

Jose, A.M. and Koelle, M.R. 2005. Domains, amino acid residues, and new isoforms of Caenorhabditis elegans diacylglycerol kinase 1 (DGK-1) important for terminating diacylglycerol signaling in vivo. J. Biol. Chem. 280: 2730-2736.

Kazanietz, M.G., Wang, S., Milne, G.W., Lewin, N.E., Liu, H.L., and Blumberg, P.M. 1995. Residues in the second cysteinerich region of protein kinase $\mathrm{C} \delta$ relevant to phorbol ester binding as revealed by site-directed mutagenesis. I. Biol. Chem. 270: 21852-21859.

Kimura, K., Ito, M., Amano, M., Chihara, K., Fukata, Y., Nakafuku, M., Yamamori, B., Feng, J., Nakano, T., Okawa, K., et al. 1996. Regulation of myosin phosphatase by Rho and Rhoassociated kinase (Rho-kinase). Science 273: 245-248.

Kowluru, A., Li, G., Rabaglia, M.E., Segu, V.B., Hofmann, F., Aktories, K., and Metz, S.A. 1997. Evidence for differential roles of the Rho subfamily of GTP-binding proteins in glucose- and calcium-induced insulin secretion from pancreatic $\beta$ cells. Biochem. Pharmacol. 54: 1097-1108.

Kuromi, H. and Kidokoro, Y. 1998. Two distinct pools of synaptic vesicles in single presynaptic boutons in a temperature-sensitive Drosophila mutant, shibire. Neuron 20: 917925.

Lackner, M.R., Nurrish, S.J., and Kaplan, J.M. 1999. Facilitation of synaptic transmission by EGL-30 Gq $\alpha$ and EGL-8 PLC $\beta$ : DAG binding to UNC-13 is required to stimulate acetylcholine release. Neuron 24: 335-346.

Los, A.P., van Baal, J., de Widt, J., Divecha, N., and van Blitterswijk, W.J. 2004. Structure-activity relationship of diacylglycerol kinase $\theta$. Biochim. Biophys. Acta 1636: 169-174.

Luo, B., Regier, D.S., Prescott, S.M., and Topham, M.K. 2004. Diacylglycerol kinases. Cell Signal. 16: 983-989.

Maesaki, R., Ihara, K., Shimizu, T., Kuroda, S., Kaibuchi, K., and Hakoshima, T. 1999. The structural basis of Rho effector recognition revealed by the crystal structure of human RhoA complexed with the effector domain of PKN/PRK1. Mol. Cell 4: 793-803.

Mendel, J.E., Korswagen, H.C., Liu, K.S., Hajdu-Cronin, Y.M., Simon, M.I., Plasterk, R.H., and Sternberg, P.W. 1995. Participation of the protein Go in multiple aspects of behavior in C. elegans. Science 267: 1652-1655.

Meng, Y., Zhang, Y., Tregoubov, V., Janus, C., Cruz, L., Jackson, M., Lu, W.Y., MacDonald, J.F., Wang, J.Y., Falls, D.L., et al. 2002. Abnormal spine morphology and enhanced LTP in LIMK-1 knockout mice. Neuron 35: 121-133.

Meng, Y., Takahashi, H., Meng, J., Zhang, Y., Lu, G., Asrar, S., Nakamura, T., and Jia, Z. 2004. Regulation of ADF/cofilin phosphorylation and synaptic function by LIM-kinase. Neuropharmacology 47: 746-754.

Miller, R.J. 1998. Presynaptic receptors. Annu. Rev. Pharmacol. Toxicol. 38: 201-227.

Miller, K.G., Alfonso, A., Nguyen, M., Crowell, J.A., Johnson, C.D., and Rand, J.B. 1996. A genetic selection for Caenorhabditis elegans synaptic transmission mutants. Proc. Nat1. Acad. Sci. 93: 12593-12598.

Miller, K.G., Emerson, M.D., and Rand, J.B. 1999. Go $\alpha$ and dia- 
cylglycerol kinase negatively regulate the $\mathrm{Gq} \alpha$ pathway in $C$. elegans. Neuron 24: 323-333.

Mochida, S., Nonomura, Y., and Kobayashi, H. 1994. Analysis of the mechanism for acetylcholine release at the synapse formed between rat sympathetic neurons in culture. $\mathrm{Mi}$ crosc. Res. Tech. 29: 94-102.

Morales, M., Colicos, M.A., and Goda, Y. 2000. Actin-dependent regulation of neurotransmitter release at central synapses. Neuron 27: 539-550.

Nguyen, M., Alfonso, A., Johnson, C.D., and Rand, J.B. 1995. Caenorhabditis elegans mutants resistant to inhibitors of acetylcholinesterase. Genetics 140: 527-535.

Nonet, M.L., Grundahl, K., Meyer, B.J., and Rand, J.B. 1993. Synaptic function is impaired but not eliminated in C. elegans mutants lacking synaptotagmin. Cell 73: 1291-1305.

Nurrish, S., Segalat, L., and Kaplan, J.M. 1999. Serotonin inhibition of synaptic transmission: $\mathrm{G} \alpha(0)$ decreases the abundance of UNC-13 at release sites. Neuron 24: 231-242.

Ramakers, G.J. 2002. Rho proteins, mental retardation and the cellular basis of cognition. Trends Neurosci. 25: 191-199.

Rhee, J.S., Betz, A., Pyott, S., Reim, K., Varoqueaux, F., Augustin, I., Hesse, D., Sudhof, T.C., Takahashi, M., Rosenmund, C., et al. 2002. $\beta$ Phorbol ester- and diacylglycerol-induced augmentation of transmitter release is mediated by Munc13s and not by PKCs. Cell 108: 121-133.

Richmond, J.E., Davis, W.S., and Jorgensen, E.M. 1999. UNC-13 is required for synaptic vesicle fusion in C. elegans. Nat. Neurosci. 2: 959-964.

Richmond, J.E., Weimer, R.M., and Jorgensen, E.M. 2001. An open form of syntaxin bypasses the requirement for UNC-13 in vesicle priming. Nature 412: 338-341.

Sahai, E., Alberts, A.S., and Treisman, R. 1998. RhoA effector mutants reveal distinct effector pathways for cytoskeletal reorganization, SRF activation and transformation. EMBO $J$. 17: 1350-1361.

Sakane, F. and Kanoh, H. 1997. Molecules in focus: Diacylglycerol kinase. Int. J. Biochem. Cell. Biol. 29: 1139-1143.

Segalat, L., Elkes, D.A., and Kaplan, J.M. 1995. Modulation of serotonin-controlled behaviors by Go in Caenorhabditis elegans. Science 267: 1648-1651.

Shupliakov, O., Bloom, O., Gustafsson, J.S., Kjaerulff, O., Low, P., Tomilin, N., Pieribone, V.A., Greengard, P., and Brodin, L. 2002. Impaired recycling of synaptic vesicles after acute perturbation of the presynaptic actin cytoskeleton. Proc. Natl. Acad. Sci. 99: 14476-14481.

Silinsky, E.M. and Searl, T.J. 2003. Phorbol esters and neurotransmitter release: More than just protein kinase C? Br. J. Pharmacol. 138: 1191-1201.

Soderling, S.H., Langeberg, L.K., Soderling, J.A., Davee, S.M., Simerly, R., Raber, J., and Scott, J.D. 2003. Loss of WAVE-1 causes sensorimotor retardation and reduced learning and memory in mice. Proc. Natl. Acad. Sci. 100: 1723-1728.

Spencer, A.G., Orita, S., Malone, C.J., and Han, M. 2001. A RHO GTPase-mediated pathway is required during P cell migration in Caenorhabditis elegans. Proc. Nat1. Acad. Sci. 98: 13132-13137.

Sudhof, T.C. 2004. The synaptic vesicle cycle. Annu. Rev. Neurosci. 27: 509-547.

Udo, H., Jin, I., Kim, J.-H., Li, H.-L., Youn, T., Hawkins, R.D., Kandel, E.R., and Bailey, C.H. 2005. Serotonin-induced regulation of the actin network for learning-related synaptic growth requires Cdc42, N-WASP, and PAK in aplysia sensory neurons. Neuron 45: 887.

van Blitterswijk, W.J. and Houssa, B. 2000. Properties and functions of diacylglycerol kinases. Cell Signal. 12: 595-605.

Wang, L., Li, G., and Sugita, S. 2004. RalA-exocyst inter- action mediates GTP-dependent exocytosis. J. Biol. Chem. 279: 19875-19881.

Wang, H.G., Lu, F.M., Jin, I., Udo, H., Kandel, E.R., de Vente, J., Walter, U., Lohmann, S.M., Hawkins, R.D., and Antonova, I. 2005. Presynaptic and postsynaptic roles of NO, cGK, and RhoA in long-lasting potentiation and aggregation of synaptic proteins. Neuron 45: 389-403.

Weston, C., Gordon, C., Teressa, G., Hod, E., Ren, X.D., and Prives, J. 2003. Cooperative regulation by Rac and Rho of agrin-induced acetylcholine receptor clustering in muscle cells. J. Biol. Chem. 278: 6450-6455.

Zallen, J.A., Peckol, E.L., Tobin, D.M., and Bargmann, C.I. 2000. Neuronal cell shape and neurite initiation are regulated by the Ndr kinase SAX-1, a member of the Orb6/COT-1/warts serine/threonine kinase family. Mol. Biol. Cell 11:31773190.

Zhang, G., Kazanietz, M.G., Blumberg, P.M., and Hurley, J.H. 1995. Crystal structure of the cys2 activator-binding domain of protein kinase $\mathrm{C} \delta$ in complex with phorbol ester. Cell 81: 917-924. 


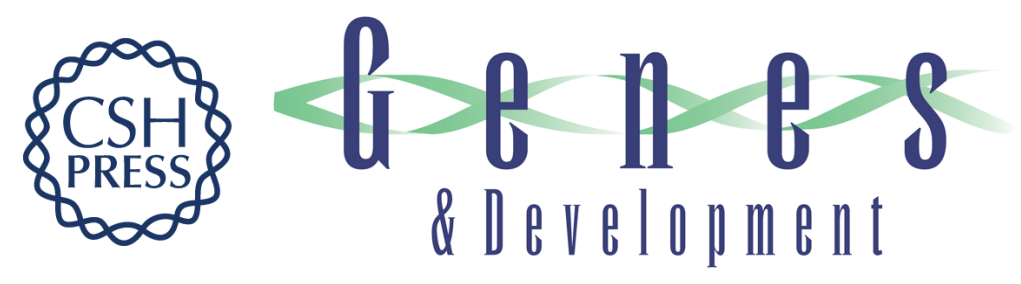

\section{Rho is a presynaptic activator of neurotransmitter release at pre-existing synapses in $C$. elegans}

Rachel McMullan, Emma Hiley, Paul Morrison, et al.

Genes Dev. 2006, 20:

Access the most recent version at doi:10.1101/gad.359706

Supplemental http://genesdev.cshlp.org/content/suppl/2005/12/30/20.1.65.DC1
Material

References This article cites 61 articles, 27 of which can be accessed free at: http://genesdev.cshlp.org/content/20/1/65.full.html\#ref-list-1

License

Email Alerting

Receive free email alerts when new articles cite this article - sign up in the box at the top Service right corner of the article or click here.

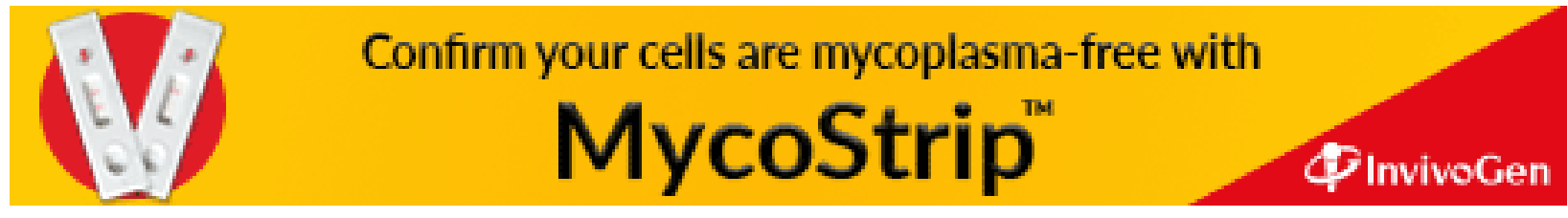

